\title{
Nouveaux concepts sur la régulation de la sécrétion d'aldostérone ; interactions endocrines, paracrines, autocrines et neurocrines
}

Les travaux des dernières années montrent de plus en plus que la régulation de la sécrétion d'aldostérone par la zone glomérulée du cortex surrénalien serait contrôlée de façon autocrine et paracrine plutôt que par la voie systémique générale. Cette hypothèse repose sur : (1) la mise en évidence de la production locale d'angiotensine II ; (2) la démonstration du rôle de l'endothélium vasculaire ; (3) l'importance de l'innervation locale ; et (4) la reconsidération des relations medulla-cortex. Ces régulations locales pourraient expliquer, soit le maintien d'une sécrétion normale d'aldostérone lors de dérèglements périphériques de plusieurs facteurs dits régulateurs (comme l'angiotensine II), soit, au contraire, l'augmentation de sécrétion, sans modification des niveaux périphériques d'angiotensine II, observée, par exemple, dans le phéochromocytome et le syndrome de Cushing.

\section{ADRESSE ET TIRÉS À PART}

N. Gallo-Payet : professeur. Service d'endocrinologie, département de Médecine, faculté de médecine, université de Sherbrooke, Sherbrooke, Québec, JIH 5N4 Canada. $m / s n^{\circ} 8-9$ vol. 9 , août-septembre 93 a glande surrénale est composée de deux parties bien distinctes du point de vue aussi bien embryologique et histologique que fonctionnel. Les cellules de la glande corticosurrénale ont une origine mésodermique alors que les cellules de la médullosurrénale ont une origine nerveuse, neuro-ectodermique. La corticosurrénale est formée de trois zones histologiques distinctes. La zone glomérulée, la plus externe et la plus étroite, est responsable de la synthèse et de la sécrétion des minéralocorticoïdes, dont le principal est l'aldostérone. La zone fasciculée, la plus large, synthé- tise les glucocorticoïdes (le principal étant le cortisol chez l'homme et le bœuf, la corticostérone chez le rat) et, à un moindre degré, des androgènes. La zone réticulée, au contact de la medulla, sécrète seulement les androgènes. La biosynthèse de ces hormones stéroïdes passe par une série d'intermédiaires dont le précurseur est le cholestérol. Les cellules médullaires, ou chromaffines, sécrètent deux catécholamines importantes, l'adrénaline et la noradrénaline. La synthèse de l'adrénaline est contrôlée principalement par les glucocorticoïdes du cortex, dont les actions s'exercent par un système porte corticosurrénalien. 


\section{RÉFÉRENCES}

1. Engleland WC, Gann DS. Splanchnic nerve stimulation modulates steroid secretion in hypophysectomized dogs. Neuroendocrinology $1987 ; 50: 124-31$

2. Bird IM, Walker SW, Williams BC. Agonist-stimulated turnover of the phosphoinositides and the regulation of adrenocortical steroidogenesis. $J$ Mol Endocrinol 1990 ; 5 : 191-209.

3. Quinn SJ, Williams GH. Regulation of aldosterone secretion. In : James VHT, ed. The Adrenal Gland, 2nd ed. New York Raven Press, 1992 : 159-89.

4. Durroux T, Gallo-Payet N, Payet MD. Effects of adrenocorticotropin on action potential and calcium currents in cultured rat and bovine glomerulosa cells. Endocrino$\log y 1991 ; 129: 2139-47$

5. Gallo-Payet N, Chouinard I, Balestre $\mathrm{MN}$, el al. Mechanisms involved in the interaction of dopamine with angiotensin II on aldosterone secretion in isolated and cultured rat adrenal glomerulosa cells. Mol Cell Endocrinol 1991; 81 : 11-23.

6. Bird IM, Nicol M, Williams BC, et al. Vasopressin stimulates cortisol secretion and phosphoinositide catabolism in cultured bovine adrenal fasciculata/reticularis cells. J Mol Endocrinal 1990; 5 : 109-16.

7. Payet $\mathrm{N}$, Isler $\mathrm{H}$. Adrenal glomerulosa mitotic stimulation by posterior pituitary hormones. Cell Tissue Res 1976; 172 : 93-101.

8. Payet N, Lehoux JG. A comparative study of the role of vasopressin and ACTH in the regulation of growth and function of rat adrenal glands. J Steroid Biochem 1980 ; $12: 462-7$

9. Gallo-Payet N, Balestre MN, Chouinard I, et al. Involvement of distinct $\mathrm{G}^{-}$ proteins in the action of vasopressin on rat glomerulosa cells. Endocrinology $1990 ; 126$ : 1699-708.

10. Wittert GA, Crock PA, Donald RA, et al. Arginine vasopressin in Cushing's disease. Lancet 1990; 35 : 991-4.

11. Tannahill LA, Dow RC, Fairhall KM, et al. Comparison of adrenocorticotropin control in Brattleboro, Long Evans and Wistar rats. Neuroendocrinology $1988 ; 8: 650-70$.

12. Kapas S, Orford CD, Barkker GP, et al. Studies on the intracellular mechanism of action of $\alpha$-melanocyte-stimulating hormone on rat adrenal zona glomerulosa. J Mol Endocrinol $1992 ; 9$ : 47-54.

13. Pham-Huu-Trung MT, Bogyo A, De Smitter N, et al. Effects of proopiomelanocortin peptides and angiotensin II on steroidogenesis in isolated aldosteronoma cells.
Les deux stimuli essentiels de la sécrétion d'aldostérone sont l'angiotensine II et une élévation de potassium $\left(\mathrm{K}^{+}\right)$, alors que le contrôle de la sécrétion de cortisol est assuré par l'hormone d'origine hypophysaire, l'adrénocorticotrophine (ACTH). Tels sont les préceptes concernant la régulation des stéroïdes surrénaliens. De faibles variations des niveaux plasmatiques de ces régulateurs entraînent des variations importantes dans la sécrétion de ces stéroïdes. Pourtant, plusieurs observations montrent que, dans certaines conditions pathologiques, l'élévation de cortisol n'est pas toujours associée à une augmentation d'ACTH. Certains travaux ont en effet montré qu'une stimulation électrique du nerf splanchnique augmentait la sensibilité du cortex à l'ACTH alors qu'une dénervation la diminuait [1]. De plus, les études réalisées in vitro, sur cellules isolées, montrent que plusieurs hormones, autres que l'ACTH et l'angiotensine II, et plusieurs neurotransmetteurs peuvent soit stimuler, soit inhiber la sécrétion d'aldostérone (figure 1). Le rôle physiologique de ces substances a toujours été mis en doute à cause des concentrations élevées nécessaires à leur action, alors que leur concentration dans la circulation générale est faible. Ce bref aperçu nous montre que la sécrétion d'aldostérone est contrôlée de façon autocrine (production d'angiotensine II), paracrine (endothélium vasculaire, tissu médullaire) et nerveuse (innervation directe).

\section{Les facteurs qualifiés d'essentiels}

L'angiotensine II est le principal stimulus physiologique de la sécrétion d'aldostérone, mais également de la sécrétion de cortisol par les cellules fasciculées bovines [2]. Inositolphosphates, diacylglycérol et calcium sont les principaux seconds messagers mis en jeu à la suite de la liaison de l'angiotensine II à son récepteur de type AT 1 [2, 3]. Bien que l'ion $\mathrm{K}^{+}$ne soit pas un stimulus physiologique, les cellules glomérulées sont très sensibles in vitro à ses variations, pour des concentrations allant de 3 à $10 \mathrm{mM}$. L'élévation du $\mathrm{K}^{+}$, en dépolarisant la membrane, permet aux canaux dépendants du voltage de s'ouvrir, entraînant une augmentation de la concentration intracellulaire de calcium [3]. Chez le rat, l'ACTH est, in vitro, le plus puissant sécrétagogue connu, alors que chez l'homme, les variations de sécrétion de cette hormone affectent peu la sécrétion

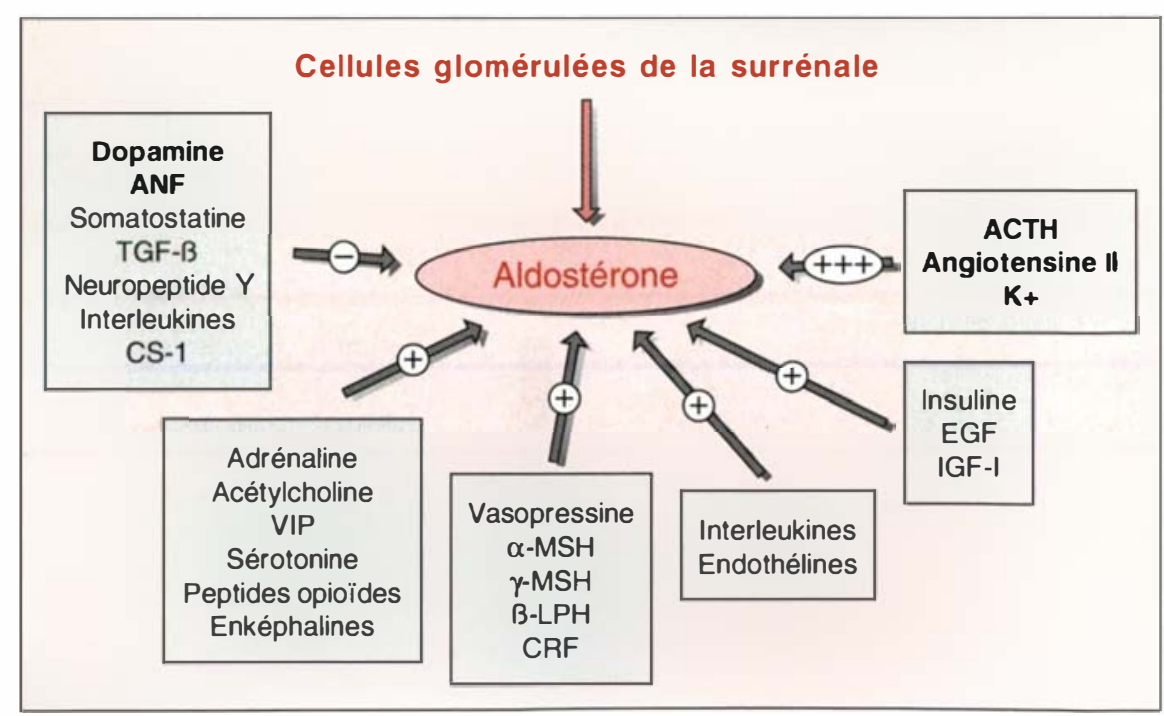

Figure 1. Les principaux facteurs agissant sur la sécrétion d'aldostérone. ACTH : hormone corticotrope ; $\mathrm{K}^{+}$: ion potassium ; EGF : facteur de croissance épidermique ; IGF-1 : facteur de croissance insulinique ; MSH : hormone mélanostimulante ; $L P H$, hormone lipotrophique ; CRF : facteur de libération de l'hormone corticotrope; VIP : peptide intestinal vaso-actif; ANF : facteur natriurétique cardiaque; TGF : facteur de croissance transformant ; CS : corticostatine. 
d'aldostérone [3]. AMPcyclique (AMPc) et calcium agissent en étroite synergie pour stimuler la sécrétion d'aldostérone [4]. Le facteur natriurétique cardiaque (ANF) diminue la sécrétion d'aldostérone induite par l'ACTH ou l'angiotensine II. Cet effet serait assuré à la fois par une phosphodiestérase sensible au GMPc et par une action directe sur les canaux calciques [3]. La dopamine (DA) inhibe la sécrétion d'aldostérone induite par l'angiotensine II. Cette action s'exerce par l'intermédiaire de récepteurs de types $\mathrm{DA}_{2}$ qui diminuent à la fois la production d'inositolphosphates et l'influx calcique stimulés par l'angiotensine II [5]. Les cellules glomérulées possèdent aussi des récepteurs de type $\mathrm{DA}_{1}$ qui stimulent la production d'AMPc associée à la sécrétion d'aldostérone [5]. Selon les niveaux d'expression de ces deux sous-types de récepteurs, la dopamine peut donc inhiber, stimuler ou ne pas modifier la sécrétion d'aldostérone induite par l'angiotensine II.

\section{Régulation paracrine ou systémique?}

Après plusieurs années de controverse, il est maintenant bien établi que la vasopressine fait partie de la longue liste des hormones participant à la régulation de la sécrétion, non seulement de l'aldostérone [2, 3], mais aussi du cortisol [6]. Nous avons été parmi les premiers à montrer que la vasopressine agissait spécifiquement sur la croissance de la zone glomérulée et sur la sécrétion d'aldostérone [7, 8]. Cette action se manifeste après liaison à des récepteurs de type V1a, et s'accompagne d'une production d'inositolphosphates par l'intermédiaire d'une protéine $G$ insensible aux toxines de choléra ou de pertussis. En revanche, l'influx calcique stimulé par la vasopressine est sensible à la toxine de $B$. pertussis, et cet influx est nécessaire au maintien de la production d'inositolphosphates [9]. Nos études récentes montrent, de plus, que la vasopressine stimule non seulement la sécrétion d'aldostérone, mais aussi la sécrétion de cortisol dans les cellules corticales de surrénale humaine. Les mécanismes de transduction utilisés $\mathrm{m} / \mathrm{s} n^{\circ} 8-9$ vol. 9, août-seplembre 93 sont identiques à ceux décrits chez le rat. L'importance physiologique de cette régulation est plus difficile à établir chez l'homme. En effet, cette hormone participe, avec le facteur de libération adrénocorticotrophique $(\mathrm{CRF})$, à la libération de l'ACTH hypophysaire ; il n'est donc pas facile d'évaluer un effet direct, indépendant de son rôle adénohypophysaire. Cependant, dans certaines situations physiologiques particulières, comme la maladie de Cushing [10], la vasopressine potentialise l'effet de l'ACTH sur la sécrétion de cortisol. Chez les patients atteints de diabète insipide neurogénique, on observe peu ou pas de variation des niveaux d'aldostérone, ce qui suggère que le rôle de la vasopressine dans la régulation cortico-surrénalienne est mineur. Pourtant, des études réalisées chez le rat Long Evans-Brattleboro (rat homozygote pour le diabète insipide) montrent que la quantité de vasopressine synthétisée au niveau de la medulla est augmentée ; la vasopressine pourrait agir de façon paracrine pour suppléer à l'absence hypophysaire [11]. Plusieurs dérivés de la proopiomélanocortine peuvent stimuler la sécrétion d'aldostérone, en particulier l'hormone mélanostimulante $(\alpha$ $\mathrm{MSH})$, la $\beta$-lipotropine (LPH), mais aussi la $\gamma$-MSH et la $\beta$-endorphine [12]. Ces effets ont été observés aussi bien chez l'homme que dans les modèles animaux. De plus, ces peptides sont tous présents dans la médullosurrénale et pourraient moduler la sécrétion d'aldostérone, en particulier lors de la genèse d'un aldostéronisme primaire [13]. Le complexe CRF-ACTH a été identifié au niveau de la médullosurrénale et les travaux récents du groupe de Van Oers [14] ont montré que le CRF d'origine médullaire pouvait être sécrété après stimulation électrique du nerf splanchnique. Cette libération augmenterait la sensibilité du cortex à l'ACTH. Le (ou les) mécanisme(s) impliqué(s) dans cette régulation reste(nt) à démontrer.

\section{Régulation paracrine}

Les catécholamines de la médullosurrénale, adrénaline et noradrénaline, stimulent la sécrétion d'aldostérone en empruntant la voie de
l'AMPc. Ces effets se manifestent surtout dans les cellules en culture [5, 15]. Cette particularité tient au fait que les cellules fraîchement préparées ont un niveau basal de sécrétion de stéroïdes très élevé, ce qui les rend moins sensibles à leurs stimuli que les cellules en culture. Plusieurs autres peptides ou neurotransmetteurs, identifiés dans la médullosurrénale, stimulent aussi bien la sécrétion d'aldostérone que celle du cortisol, particulièrement dans des situations de stress. Parmi ceux-ci on trouve l'acétylcholine [16], le peptide vaso-actif intestinal (VIP) [17], la galanine et les enképhalines. Leurs actions s'exercent via une augmentation d'AMPc. Au contraire, le neuropeptide $\mathrm{Y}$ et la somatostatine (SRIF) [18] inhibent la stimulation de la sécrétion d'aldostérone induite par l'angiotensine II, le $\mathrm{K}^{+}$et l'ACTH. Cette inhibition serait due à l'activation d'une protéine de type $\mathrm{G}_{\mathrm{i}}$.

Certains facteurs de croissance, en particulier l'insuline [19], l'IGF-I [20] et le facteur de croissance épidermique (EGF) [21], maintiennent les fonctions sécrétoires du cortex surrénalien et sont actifs sur la trophicité tissulaire. Ces facteurs non seulement stimulent la prolifération des cellules glomérulées, mais aussi potentialisent les effets de l'angiotensine II et de l'ACTH $[19,21]$. La présence d'insuline est indispensable au maintien des fonctions stéroïdogéniques des cellules en culture, tant glomérulées que fasciculées, qu'elles soient d'origine murine, bovine ou humaine [20]. Les récepteurs des facteurs de croissance possèdent une activité tyrosine kinase au niveau de leur domaine cytoplasmique. L'activation du récepteur, et l'autophosphorylation qui en résulte, leur permet de s'associer à d'autres protéines participant au transfert des signaux mitogéniques [22, 23]. Les cellules glomérulées sont le siège d'une activité mitotique importante, supportant le concept qu'après division, une partie de ces cellules migreraient pour donner les cellules fasciculées/réticulées [24]. Le degré de différenciation des cellules au stade glomérulé semble dépendre de l'interaction entre plusieurs facteurs, puisque, mises en culture cellulaire prolongée, les cellules glomérulées acquièrent le phénotype fasciculé [25]. 


\section{RÉFÉRENCES}

14. Van Oers JW, Hinson JP, Binnekade R, et al. Physiological role of corticotropin-releasing factor in the control of adrenocorticotropin-mediated corticosterone release from the rat adrenal gland. Endocrinology 1992 ; 130 : 282-8.

15. De Léan A, Racz K, NcNicoll N, et al. Direct $\beta$-adrenergic stimulation of aldosterone secretion in cultured bovine adrenal subcapsular cells. Endocrinology $1984 ; 115$ : 485-92.

16. Hadjian A, Culty J, Chambaz EM. Stimulation of phosphatidylinositol turnover by acetylcholine, angiotensin II and ACTH in bovine adrenal fasciculata cells. Biochim Biophys Acta 1984 ; 804 : 427-33.

17. Holzwarth MA, Cunningham LA, Kleitman $\mathrm{N}$. The role of adrenal nerves in the regulation of adrenocortical functions. Ann NY Acad Sci 1987 ; 512 : 449-63.

18. Hausdorff WP, Aguilera G, Catt K Inhibitory actions of somatostatin on cyclic AMP and aldosterone production in agoniststimulated adrenal glomerulosa cells. Cell Signal $1989 ; 1$ : 377-86.

19. Penhoat A, Chatelain PG, Jaillard C, et al. Characterization of insulin-like growth factor I and insulin receptors on cultured bovine adrenal fasciculata cells. Role of these peptides on adrenal cell function. Endocrino$\log y 1988$; 122 : 2518-25.

20. Pham-Huu-Trun MT, Villette JM, Bogyo A, et al. Effects of insulin-like growth factor I (IGF I) on enzymatic activity in human adrenocortical cells. Interactions with ACTH. J Steroid Biochem 1991 ; 39 : 903-9.

21. Natarajan R, Nadler J. Angiotensin IIinduced aldosterone synthesis is potentiated by epidermal growth factor. Endocrinology $1991 ; 128: 2285-90$

22. Filhol O, Cochet C. Le transfert des signaux mitogéniques : une affaire de particules. médecine/sciences $1990 ; 6: 980-4$.

23. Coulier F, Pizette S, Batoz M, Birnbaum D. Les récepteurs des facteurs de croissance : une famille qui s'agrandit. médecine/sciences $1992 ; 8: 811-8$

24. Roskelley CD, Baimbridge KG, Leun PCLK, et al. Divergent dffferentiation of rat adrenocortical cells is associated with an interuption of angiotensin II-mediated signal transduction. Mol Cell Endocrinol $1992 ; 89$ : 79-89.

25. Payet N, Lehoux JG. Effect of ACTH or zinc treatment on plasma aldosterone and corticosterone levels and on the in vitro steroid output from adrenocortical cells. Can J Biochem 1982 ; 11 : 1058-64.

26. Natarajan R, Nadler J. Platelet-derived growth factor is a potent inhibitor of angiotensin II-induced aldosterone synthesis. Mol Cell Endocrinol 1992 ; 83 : 57-63.

27. Lefebvre H, Contesse V, Delarue C, et al. Serotonin-induced stimulation of cortisol

is mediated through activation of a serotonin receptor subtype. Neuroscience $1992 ; 47$ : 999-1007.

28. Delarue C, Becquet D, Idres $\mathrm{S}$, et al. Serotonin synthesis in adrenochromaffin cells. Neuroscience $1992 ; 46: 495-500$.

29. Hinson GP, Vinson GP, Kapas S, et al. The role of endothelin in the control of adrenocortical function : stimulation of endothelin release by ACTH and the effects of endothelin-1 and endothelin-3 on steroidogenesis in rat and human adrenocortical cells. J Endocrinol 1991 ; 128 : 275-80.

30. Natarajan R, Ploszaj S, Horton R, et al. Tumor necrosis factor and interleukin-1 are potent inhibitors of angiotensin-II induced aldosterone synthesis. Endocrinology 1989 ; 125 : 3084-9.

31. Zhu $\mathrm{Q}$, Solomon $\mathrm{S}$. Isolation and mode of action of rabbit corticostatic (antiadrenocorticotropin) peptides. Endocrinology 1992 ; $130: 1413-23$

32. Wang $Y$, Yamaguchi $T$, FrancoSaenz R, Mulrow PJ. Regulation of renin gene expression in rat adrenal zona glomerulosa cells. Hypertension $1992 ; 20$ : 776-81.

33. Oda H, Lotshaw DP, Franco-Saenz R, et al. Local generation of angiotensin II as a mechanism of aldosterone secretion in rat adrenal capsules. Proc Soc Exp Biol Med $1991: 196: 175-7$

34. Rebuffat $\mathrm{P}, \mathrm{Malendowicz}$ LK, Kasprzak A, et al. A coupled morphological and biochemical study on the cellular localization of the intra-adrenal renin granules in rats. Cytobios 1991; 68: 7-13.

35. Hotta M, Baird A. Differential effects of transforming growth factor type $\beta$ on the growth and function of adrenocortical cells in vitro. Proc Natl Acad Sci USA 1986; 83 : 7795-9.

36. Fei je JJ, Baird A. La crinopexie ; un modèle décrivant les mécanismes qui régissent la biodisponibilité des facteurs de croissance. médecine/sciences $1992 ; 8: 805-10$.

37. Schweigerer L, Neufeld G, Friedman J, et al. Basic fibroblast growth factor : production and growth stimulation in cultured adrenal cortex cells. Endocrinology 1987 ; $120: 796-800$

38. Grothe C, Unsicker K. Immunocytochemical localization of basic fibroblast growth in bovine adrenal gland, ovary and pituitary. J Histochem Cytochem 1989; 12 : 1877-83.

39. Charlton BG, McGadey JM, Russell D, et al. Noradrenergic innervation of the human adrenal cortex as revealed by dopamine-beta-hydroxylase immunohistochemistry. J Endocrinol 1992 ; 126 : 5-8.

40. Ang VJY, Jenkins JS. Neurohypophysial hormones in the adrenal medulla. J Clin Endocrinol Metab 1984; 58 : 688-91.
Des facteurs d'origine vasculaire agissent sur la sécrétion d'aldostérone, en particulier le facteur de croissance plaquettaire (PDGF) ; c'est un mitogène très actif dans plusieurs tissus, mais il inhibe la sécrétion d'aldostérone [26]. Bien que l'origine exacte du PDGF ne soit pas clairement élucidée, il semble bien que les plaquettes et les vaisseaux soient une source importante de ce facteur. La sérotonine est un puissant stimulant de la sécrétion d'aldostérone, aussi bien chez le rat que chez l'homme [27]. Elle agit par l'intermédiaire de récepteurs de type $5 \mathrm{HT}_{4}$, dont l'activation est associée à une augmentation d'AMPc et à un influx calcique. La sérotonine serait libérée des mastocytes présents à la périphérie cellulaire et vasculaire [27], mais elle est aussi contenue dans les neurones sérotoninergiques de la medulla [28]. Les endothélines de types 2 et 3 , elles aussi, activent la sécrétion d'aldostérone pour des concentrations aussi faibles que $10 \mathrm{fM}$; à la concentration de $100 \mathrm{nM}$, elles mutiplient par vingt la sécrétion d'aldostérone par les cellules glomérulées isolées [29]. L'endothéline 1 seule n'a pas d'effet, mais elle potentialise les effets stimulants de l'ACTH et de l'angiotensine II. Les effets de l'endothéline ont été décrits aussi bien chez le rat et le bœuf que chez l'homme, sur les cellules glomérulées et sur les cellules fasciculées [29].

Plusieurs facteurs du système immunitaire sont également impliqués dans la régulation de la sécrétion des corticostéroïdes, tel le facteur de nécrose tumorale (TNF) [30], inhibiteur actif de la sécrétion d'aldostérone induite par l'angiotensine II. Le rôle de l'interleukine I n'est pas encore clairement défini : certains groupes l'ont identifié comme un inhibiteur de la sécrétion d'aldostérone [30], alors que pour d'autres, il la stimule. Ces substances sont synthétisées à la fois par les macrophages et par les cellules endothéliales et musculaires lisses des parois vasculaires. Les peptides corticostatiques, en particulier le CS I, contenus dans les leucocytes granulocytaires, inhibent les sécrétions d'aldostérone et de corticostérone induites par l'ACTH [31]. Le Tableau $I$ résume la source et les effets des substances agissant sur les deux zones du cortex. 


\begin{tabular}{|c|c|c|c|c|c|c|}
\hline \multicolumn{7}{|c|}{ Tableau 1} \\
\hline $\begin{array}{l}\text { Peptide ou } \\
\text { neurotransmetteur }\end{array}$ & $\begin{array}{r}\text { Imr } \\
\text { loca } \\
\text { des ré }\end{array}$ & $\begin{array}{l}0- \\
\text { ion } \\
\text { iteurs }\end{array}$ & $\begin{array}{l}\text { Loca } \\
\text { des ré }\end{array}$ & $\begin{array}{l}\text { ion } \\
\text { teurs }\end{array}$ & & Effets \\
\hline Angiotensine II & $\begin{array}{l}\text { glomérulée } \\
\text { (rénine) }\end{array}$ & {$[32,33]$} & $\begin{array}{l}\text { glomérulée } \\
\text { fasciculée }\end{array}$ & [2] & 厂 & $\begin{array}{l}\text { aldostérone } \\
\text { cortisol }\end{array}$ \\
\hline Dérivés du POMC & medulla & [43] & $\begin{array}{l}\text { glomérulée } \\
\text { fasciculée }\end{array}$ & [1] & I & $\begin{array}{l}\text { aldostérone } \\
\text { cortisol }\end{array}$ \\
\hline Vasopressine & medulla & {$[40,52]$} & $\begin{array}{l}\text { glomérulée } \\
\text { fasciculée }\end{array}$ & $\begin{array}{l}{[2]} \\
{[2]}\end{array}$ & 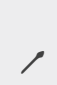 & $\begin{array}{l}\text { aldostérone } \\
\text { cortisol }\end{array}$ \\
\hline CRF-ACTH & medulla & [41] & $\begin{array}{l}\text { glomérulée } \\
\text { fasciculée }\end{array}$ & 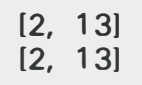 & r & $\begin{array}{l}\text { aldostérone } \\
\text { cortisol }\end{array}$ \\
\hline $\begin{array}{l}\text { Peptide vaso-actif } \\
\text { intestinal (VIP) }\end{array}$ & $\begin{array}{l}\text { medulla } \\
\text { glomérulée }\end{array}$ & [17] & glomérulée & [17] & l & aldostérone \\
\hline Dopamine & $\begin{array}{l}\text { medulla } \\
\text { glomérulée }\end{array}$ & {$[45]$} & glomérulée & [2] & 1 & $\begin{array}{l}\text { aldostérone } \\
\text { induit } \\
\text { par A II }\end{array}$ \\
\hline $\begin{array}{l}\text { Facteur natriurétique } \\
\text { cardiaque (ANF) }\end{array}$ & medulla & [43] & glomérulée & {$[2,43]$} & 1 & $\begin{array}{c}\text { aldostérone } \\
\text { induit } \\
\text { par A II }\end{array}$ \\
\hline $\begin{array}{l}\text { Neuropeptide } Y \\
\text { Substance } P\end{array}$ & $\begin{array}{l}\text { glomérulée } \\
\text { medulla }\end{array}$ & [44] & glomérulée & [44] & $\searrow$ & $\begin{array}{c}\text { aldostérone } \\
\text { induit } \\
\text { par A II }\end{array}$ \\
\hline Sérotonine & $\begin{array}{l}\text { medulla } \\
\text { glomérulée }\end{array}$ & $\begin{array}{l}{[28]} \\
{[27]}\end{array}$ & glomérulée & {$[28]$} & 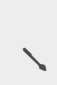 & aldostérone \\
\hline $\begin{array}{l}\text { Facteur de croissance } \\
\text { fibroblastique }\end{array}$ & $\begin{array}{l}\text { medulla } \\
\text { glomérulée }\end{array}$ & $\begin{array}{l}{[38]} \\
{[37]}\end{array}$ & glomérulée & [37] & $\searrow$ & aldostérone \\
\hline
\end{tabular}

\section{Régulation autocrine}

La zone glomérulée contient tous les composants du système rénineangiotensine. Des études d'hybridation in situ ont clairement démontré la présence d'ARNm de la rénine dans cette zone. Des cellules glomérulées en culture sont capables, à la suite de stimulations appropriées $\left(\mathrm{ACTH}, \mathrm{K}^{+}\right)$, de relâcher de la rénine dans le milieu de culture [32]. La rénine libérée permet la transformation de l'angiotensinogène en angiotensine I, puis en angiotensine II ; l'angiotensinogène et l'enzyme de conversion ont été également identifiés dans la zone glomérulée. Cette production locale d'angiotensine II serait suffisante pour contrôler la sécrétion d'aldostérone [33]. Les mécanismes de stockage et de libéra- tion de la rénine ne sont pas élucidés. Bien que les études ultrastructurales démontrent la présence de quantités variables de "corps denses" dans les cellules glomérulées [34], la présence de rénine dans ces structures n'a pas encore été démontrée.

Parmi les facteurs de croissance, le facteur de croissance transformant (TGF- $\beta$ ) apparaît comme un puissant inhibiteur de la stéroïdogenèse [35]. Les équipes de Feige et Baird ont clairement démontré que le TGF- $\beta$ était produit par les cellules corticosurrénaliennes et agirait de façon autocrine/paracrine pour moduler l'action des stimuli locaux ou périphériques [36]. Le facteur de croissance fibroblastique (FGF), comme le TGF$\beta$, est produit par les cellules corticosurrénaliennes et stimule la prolifération cellulaire de façon autocrine
[37]. Identifié dans les cellules corticales et dans la medulla [38], il pourrait donc agir à la fois de façon autocrine et paracrine.

\section{Le rôle de l'innervation dans les régulations autocrine et paracrine}

Le cortex surrénalien possède une innervation sympathique importante. Il reçoit deux types de fibres, dont les corps cellulaires sont localisés pour certains à l'extérieur de la glande et pour d'autres dans la medulla. La régulation de l'activité de libération hormonale des premières est indépendante du nerf splanchnique alors que les deuxièmes seraient contrôlées par celui-ci. Les travaux de Holzwarth [17] ont montré que la capsule et la zone glomérulée sont riches en fibres 


\section{RÉFÉRENCES}

41. Minamino $\mathrm{N}$, Uehara A, Arimura A. Biological and immunological characterization of corticotropin-releasing activity in the bovine adrenal medulla. Peptides 1988 ; 9 $37-45$.

42. Suda $T$, Tomori N, Yajima F, Odagiri E, Demura H, Shizume K. Characterization of immunoreactive corticotropin and corticotropin-releasing factor in human adrenal and ovarian tumors. Acta Endocrinol (Copenh) $1986 ; 111: 546-52$.

43. Nguyen TL, Baninski K, Ong H, et al. Differential regulation of natriuretic peptide biosynthesis in bovine adrenal chromaffin cells. Peptides $1990 ; 11$ : 973-8

44. Neri G, Andreis PG, Nussdorfer GG. Effects of neuropeptide- $Y$ and substance $P$ on the secretory activity of dispersed zonaglomerulosa cells of rat adrenal gland. Neuropeptides $1990 ; 17: 121-5$.

45. McCarty R, Kirby RF, Carey RM. Dopamine may be a neurohormone in rat adrenal cortex. Am J Physiol 1984; 247 709-13

46. Gallo-Payet $\mathrm{N}$, Pothier P, Isler $\mathrm{H}$. On the presence of chromaffin cells in the adrenal cortex : their possible role in adrenocortical function. Biochem Cell Biol 1987 ; 65 : $588-92$

47. Bornstein S, Ehrhart-Bornstein M, Usadel $\mathrm{H}$, et al. Morphological evidence for a close interaction of chromaffin cells with cortical cells within the adrenal gland. Cell Tissue Res 1991; 265 : 1-9.

48. Bornstein S, Ehrhart-Bornstein $M$ Ultrastructural evidence for a paracrine regulation of the rat adrenal cortex mediated by the local release of catecholamines from chromaffin cells. Endocrinology 1992 ; 131: 3126-8

49. Leboulanger F, Benyamina M, Delarue $\mathrm{C}$, et al. Neuronal and paracrine regulation of adrenal steroidogenesis : interactions between acetylcholine, serotonin and vasoactive intestinal peptide (VIP) on corticosteroid production by frog interrenal tissue. Brain Res $1988 ; 453$ : 103-9.

50. Hinson JP. Paracrine control of adrenocortical function: a new role for the medulla ? J Endocrinol $1989 ; 124$ : 7-9

51. O'Brien T, Young WF, Davila DG, et al. Cushing's syndrome associated with ectopic production of corticotrophin-releasing hormone, corticotrophin and vasopressin by a pheochromocytoma. Clin Endocrinol 1992 ; 37 : 460-7.

52. Perraudin V, Delarue C, Lefevre H, et al. Vasopressin stimulates cortisol secretion from human adrenocortical tissue through activation of $\mathrm{V}_{1}$ receptors. $J$ Clin Invest $1993 ; 76: 1522-8$.

53. Mazzochi G, Markowska A, Malendowicz LK, Musajo F et al. Evidence that endogenous arginine-vasopressin (AVP) is involved in the maintenance of the growth and steroidogenic capacity of rat adrenal zona glomerulosa. J Steroid Biochem Mol Biol
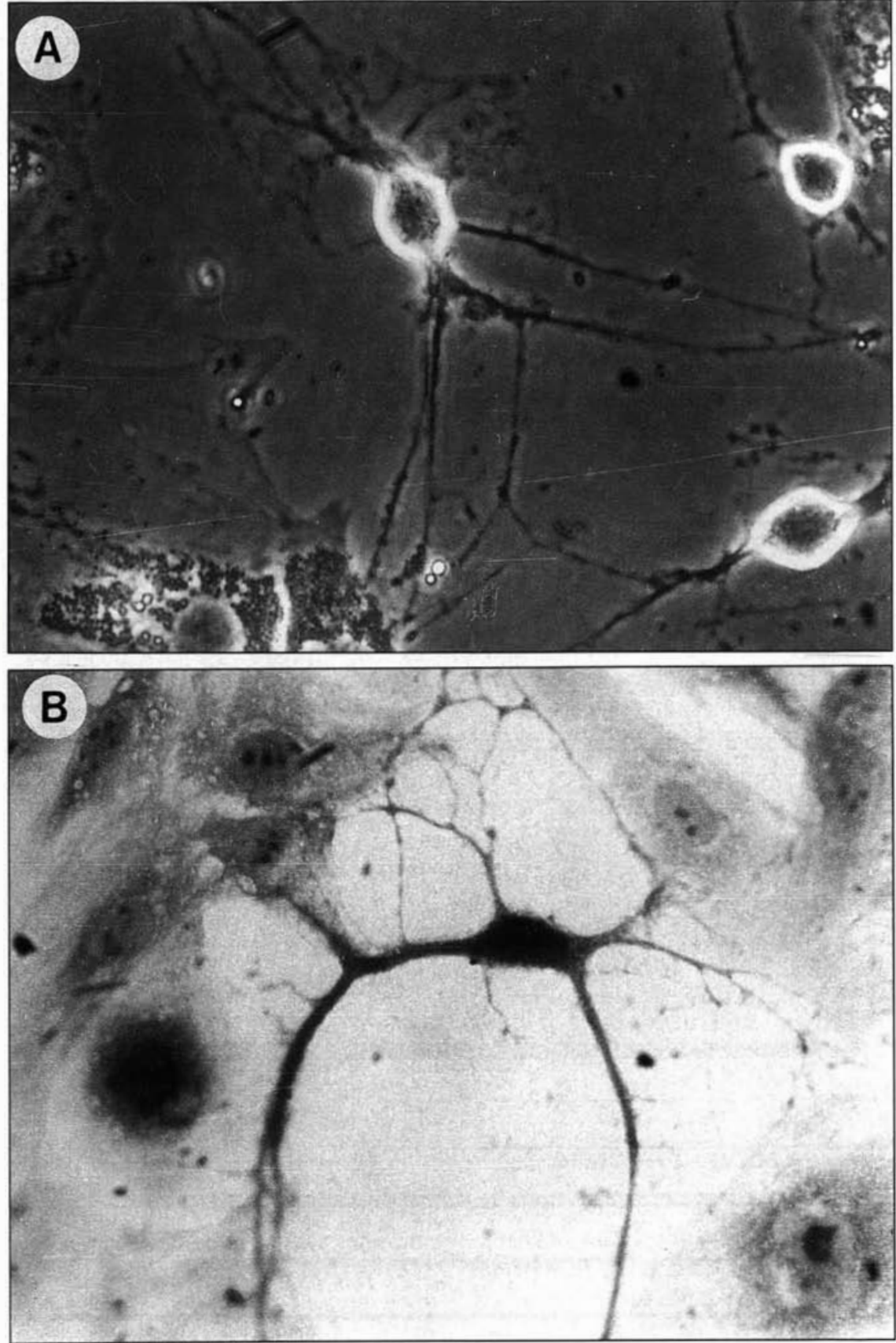

Figure 2. Une innervation directe des cellules glomérulées peut être observée en culture. Dans des cellules glomérulées cultivées pendant trois jours dans un milieu de culture complet, il est fréquent d'observer des prolongements de cellules nerveuses (au centre dans les deux photos) projetant des ramifications s'appuyant sur les cellules glomérulées (situées ici à la périphérie). Photographies prises (A) au microscope à contraste de phase et (B) en illumination normale (x 330). 


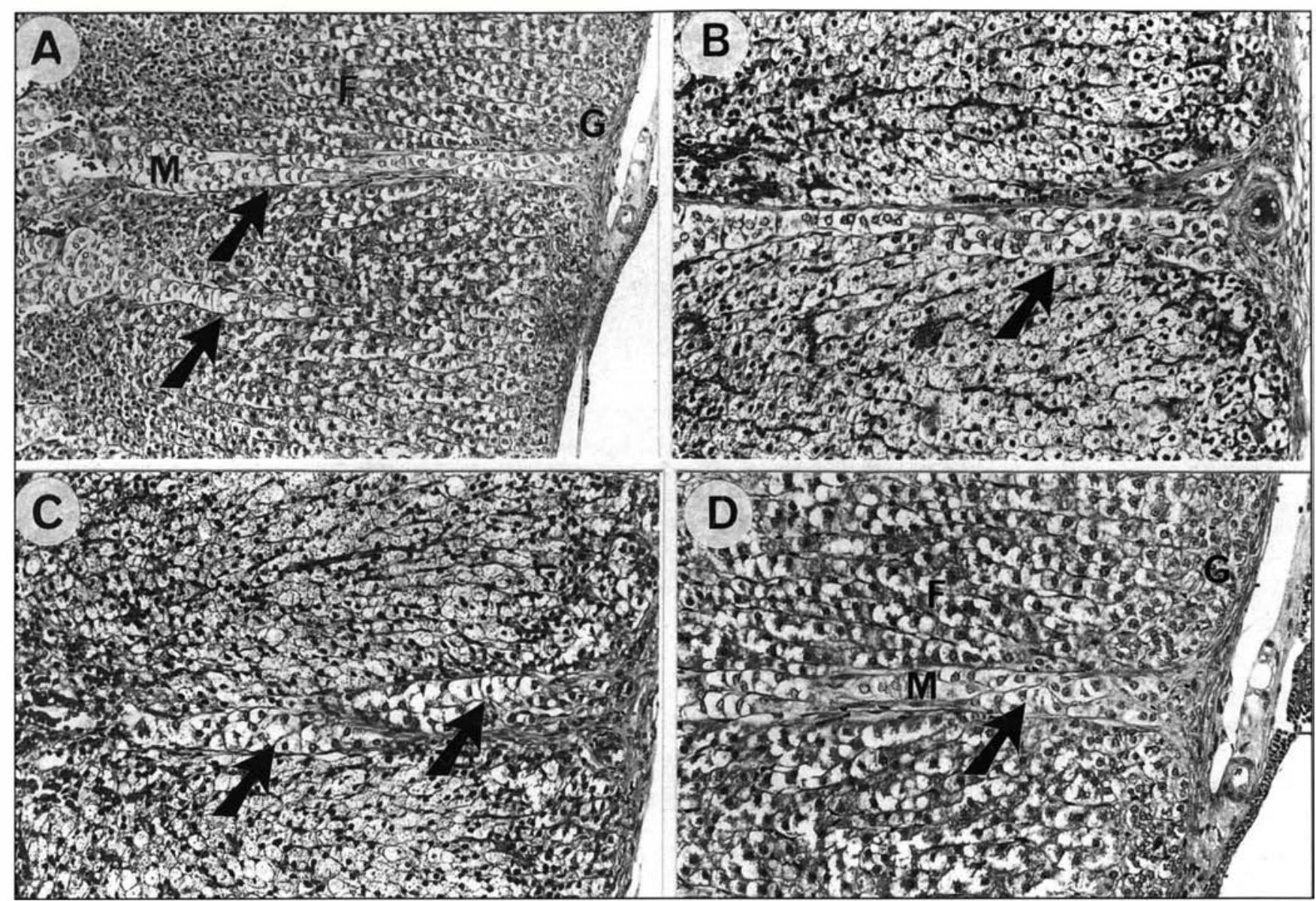

Figure 3. Présence de rayons médullaires à travers le cortex. Sur quatre sections histologiques lquadrants $A$, B, $C$ et $D$ l de surrénale de rat Long Evans, on peut observer la présence de travées médullaires traversant le cortex pour s'élargir au contact de la capsule. G : zone glomérulée, $F$ : zone fasciculée, $M$ : medulla, Les flèches indiquent l'emplacement des rayons (x 18).

catécholaminergiques, qui forment une arborisation développéc autour des vaisseaux sanguins et des glomérules cellulaircs. Des fibres VIPergiques ont été également identifiées, qui proviennent de la medulla et s'étendent à travers tout le cortex. Le VIP est même un sécrétagogue plus puissant que l'angiotensine II lorsqu'on utilise des préparations tissulaires correspondant à la capsulc et à la zone glomérulée [17]. Une innervation adrénergique a également été observée au niveau des surrénales humaines [39], et pourrait expliquer les effets de l'adrénaline et de la noradrénaline, tant sur la sécrétion d'aldostérone que sur celle de cortisol. Nous observons fréquemment, lors de la mise en culture de cellules glomérulées, la présence de fibres nerveuses élaborant des ramifications qui s'appuient sur les cellules glomérulées (figure 2). Toutes ces observam/s n० 8.9 vol. 9, août-seplembre 93 tions morphologiques étayent l'hypothèsc d'une régulation paracrine de la sécrétion d'aldostérone corticale par les ramifications nerveuses d'origine médullosurrénalienne.

\section{Les rayons médullaires}

Comme nous venons de le mentionner, la plupart des hormones peptidiques et des neurotransmetteurs agissant sur la sécrétion d'aldostérone ont été identifiés, non seulement dans la medulla mais aussi dans les zones corticales. C'est en particulier le cas de la vasopressine [40], des dérivés de la pro-opiomélanocortine [41], du CRF [42], de la sérotonine et du VIP [17], du FGF [37], de l'ANF [43], du ncuropeptide $\mathrm{Y}$, de la substance $\mathrm{P}$ [44] et de la dopamine [45]. La présence de tissu médullaire à l'intérieur du cortex surrénalien a été démontrée en microscopie photonique et électro- nique [46]. La figure 3 montre que les cellules chromaffines sont facilement identifiables par leur taille et lcur coloration plus pâle sur des coupes colorées à l'hématoxyline-ćosine. A l'échelle ultrastructurale, les rayons médullaires contiennent non seulement les cellules chromaffines (avec leurs granules de sécrétion denses aux électrons), mais aussi des fibres nerveuses; les deux structures sont en contact étroit avec les cellules corticales (reconnaissables par l'abondance des mitochondries) (figures $4 A$ et $B$ ). Ces observations ont été confirmées par Bornstein et al. [47] qui ont visualisé la libération de neurotransmetteur au voisinage immédiat des cellules glomérulées [48] (figure $4 C$ ). Une des premières interprétations de la présence de ces rayons était qu'ils pourraient être des vestiges du développement embryonnaire, puisque les cellules chromaffines doivent migrer 

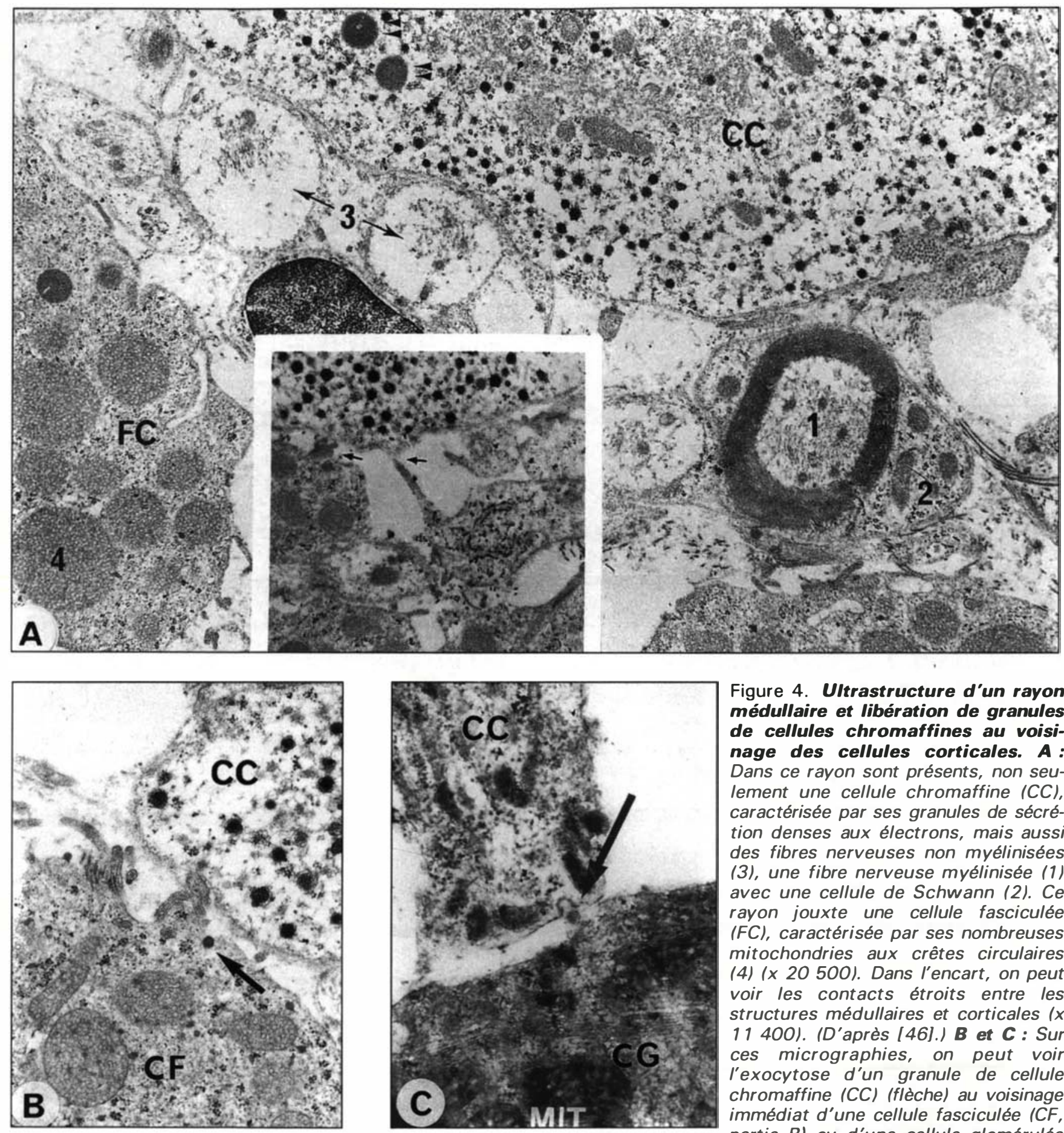

Figure 4. Ultrastructure d'un rayon médullaire et libération de granules de cellules chromaffines au voisinage des cellules corticales. A : Dans ce rayon sont présents, non seulement une cellule chromaffine (CC), caractérisée par ses granules de sécrétion denses aux électrons, mais aussi des fibres nerveuses non myélinisées (3), une fibre nerveuse myélinisée (1) avec une cellule de Schwann (2). Ce rayon jouxte une cellule fasciculée (FC), caractérisée par ses nombreuses mitochondries aux crêtes circulaires (4) (x 20 500). Dans l'encart, on peut voir les contacts étroits entre les structures médullaires et corticales $(x$ 11 400). (D'après [46].) B et C : Sur ces micrographies, on peut voir l'exocytose d'un granule de cellule chromaffine (CC) (flèche) au voisinage immédiat d'une cellule fasciculée (CF, partie B) ou d'une cellule glomérulée (CG, partie C) (x 33 000). MIT : mitochondrie. (La figure $4 \mathrm{C}$ est adaptée de la référence [47].) 


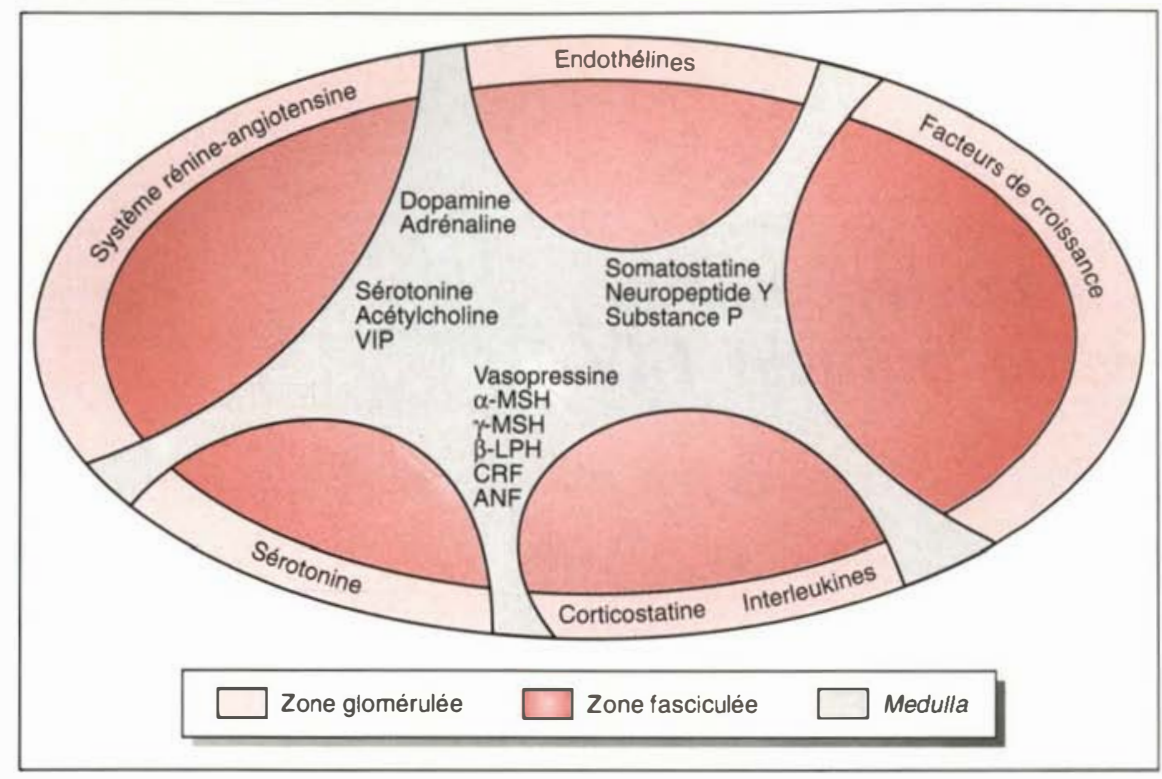

Figure 5. Régulation intrinsèque de la sécrétion d'aldostérone. Diagramme résumant l'ensemble des facteurs susceptibles de moduler les sécrétions d'aldostérone et de cortisol. Ceux-ci peuvent exercer leurs effets à travers les rayons médullaires, l'innervation médullocorticale, ou encore de façon autocrine ou paracrine.

à travers le cortex fotal pour finalement se localiser au centre de la glande. Cependant, les études récentes concernant la présence et le rôle des substances contenues dans la medulla sont plutôt en faveur d'un rôle physiologique de ces rayons dans la modulation des niveaux de sécrétion des stéroïdes des zones corticales, contrebalançant ainsi les agressions causées par la voie systémique. Les relations cellules chromaffines/cellules corticales rappellent les relations étroites existant dans le tissu interrénal de grenouille [49].

La figure 5 résume les relations anatomo-physiologiques que la medulla pourrait exercer sur le cortex, par l'intermédiaire de l'innervation médullo-surrénalienne, par le tissu médullaire présent dans le cortex, ou encore de façon autocrine ou paracrine [50]. Ces régulations locales pourraient expliquer, soit le maintien d'une sécrétion normale d'aldostérone lors de dérèglements périphériques de facteurs régulateurs dits essentiels (comme l'angiotensine II), soit, au contraire, expliquer une augmentation de sécrétion d'aldostérone, alors que les niveaux d'angiotensine II périphérique ne sont pas modifiés. Une hypertension artérielle est observée dans $80 \%$ des cas de phéochro-

\section{Summary}

New concepts for the aldosterone and cortisol regulation. Endocrine, paracrine, autocrine and neurocrine interactions

An increasing number of recent investigations reveal that the secretion of aldosterone by the zona glomerulosa and, to a lesser extent, the secretion of cortisol by the zona fasciculata may in fact be under autocrine and paracrine control rather than regulated by peripheral influences. These hypotheses are based on (1) the evidence of a local production of angiotensin II by the endogenous renin-angiotensin system, which is expressed in zona glomerulosa cells; (2) the importance of locally-induced stimulation by products of the vascular endothelium ; (3) the demonstration that local innervation stimulates the production of endogenous stimuli which in turn modulate the secretion of aldosterone and cortisol ; and (4) the presence of chromaffin cells extending into the cortex via medullary rays, which may account for the fact that many neurotransmitters and peptides of the medulla regulate steroid secretion. This local regulation may provide an explanation on the one hand for the continued normal secretion of aldosterone in conditions where peripheral stimulation by angiotensin II is altered, but also for the increases observed in aldosterone secretion, even when circulating angiotensin II concentrations remain unchanged.

\section{Note ajoutée aux épreuves}

Deux publications récentes confirment le rôle de la vasopressine dans la régulation de sécrétion de cortisol dans la surrénale humaine. Les auteurs démontrent également la présence de vasopressine dans des cellules chromaffines situées dans le cortex [52]. Une autre publication démontre également le rôle de la vasopressine endogène dans la régulation de sécrétion d'aldostérone [53]. 